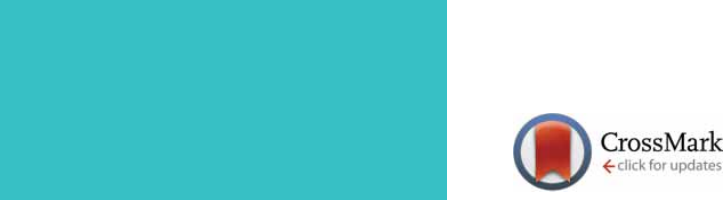

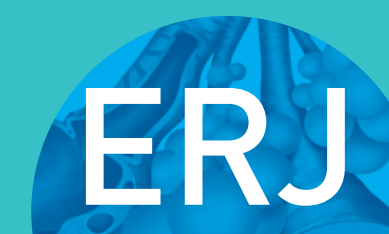

open research
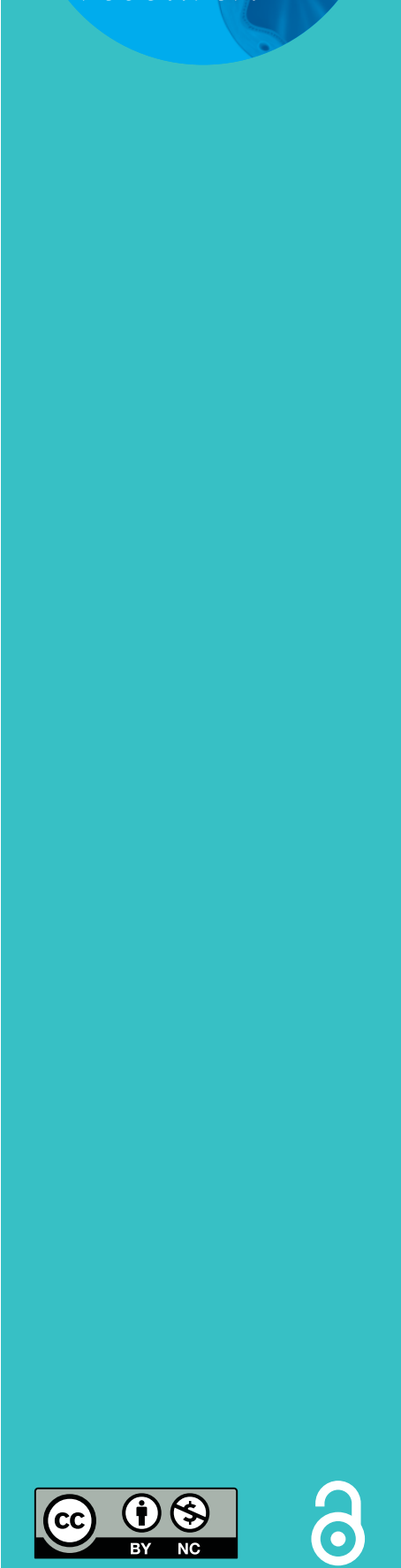

\section{Beneficial effects of adenotonsillectomy in children with sickle cell disease}

\author{
Ilaria Liguoro (1) ${ }^{1}$, Michele Arigliani (i) ${ }^{1}$, Bethany Singh ${ }^{2,3}$, Lisa Van Geyzel², \\ Subarna Chakravorty ${ }^{3}$, Cara Bossley $^{2}$, Maria Pelidis $^{4}$, David Rees ${ }^{3}$, \\ Baba P.D. Inusa $\mathbb{1}^{4}$ and Atul Gupta (10) 2,5
}

Affiliations: ${ }^{1}$ Dept of Medicine, Division of Pediatrics, University Hospital of Udine, Udine, Italy. ${ }^{2}$ Dept of Paediatric Respiratory Medicine, King's College Hospital, London, UK. ${ }^{3}$ Dept of Paediatric Haematology, King's College Hospital, London, UK. “Dept of Paediatric Haematology, Evelina London Children's Hospital, Guy's and St Thomas' NHS Trust, London, UK. 'Institute for Women's and Children's Health, King's College London, London, UK.

Correspondence: Atul Gupta, King's College Hospital, Denmark Hill, London, SE5 9RS, UK. E-mail: atul.guptaßkcl.ac.uk

ABSTRACT Tonsillectomy and adenoidectomy (T\&A) is frequently performed in children with sickle cell disease (SCD). Our aim was to evaluate the impact of this surgery on overnight oxygenation and rates of complications in these patients.

Children with SCD who underwent T\&A between 2008 and 2014 in two tertiary hospitals were retrospectively evaluated. Overnight oximetry and admission rates due to vaso-occlusive pain episodes (VOEs) and acute chest syndrome (ACS) in the year preceding and following the surgery were compared.

19 patients (10 males, 53\%) with a median age of 6 years (range 3.5-8) were included. A significant increase of mean overnight arterial oxygen saturation measured by pulse oximetry $\left(S_{\mathrm{pO}_{2}}\right)$ (from $93 \pm 3.6 \%$ to $95.3 \pm 2.8 \%, \mathrm{p}=0.001$ ), nadir $S_{\mathrm{pO}_{2}}$ (from $83.0 \pm 7.1 \%$ to $88 \pm 4.1 \%, \mathrm{p}=0.004$ ) and a reduction of $3 \%$ oxygen desaturation index (from a median value of 5.7 to $1.8, \mathrm{p}=0.003$ ) were shown. The mean annual rate of ACS decreased from $0.6 \pm 1.22$ to $0.1 \pm 0.2$ events per patient-year ( $\mathrm{p}=0.003$ ), while the mean cumulative rate of hospitalisations for all causes and the incidence of VOEs were not affected.

T\&A improved nocturnal oxygenation and was also associated with a reduction in the incidence of ACS at 1-year follow-up after surgery.

@ERSpublications

In children with SCD, this retrospective analysis suggests that tonsillectomy and adenoidectomy may have a beneficial effect on nocturnal oxygenation level and the rate of ACS. This finding will need further confirmation in prospective and larger studies. https://bit.ly/3gL6gaU

Cite this article as: Liguoro I, Arigliani M, Singh B, et al. Beneficial effects of adenotonsillectomy in children with sickle cell disease. ERJ Open Res 2020; 6: 00071-2020 [https://doi.org/10.1183/ 23120541.00071-2020].

Received: 16 Feb 2020 | Accepted after revision: 2 Aug 2020

Copyright $\odot$ ERS 2020. This article is open access and distributed under the terms of the Creative Commons Attribution Non-Commercial Licence 4.0. 


\section{Introduction}

Sickle cell disease (SCD) is the most frequent haemoglobinopathy with a global annual incidence of 300000 newborn cases [1]. SCD is characterised by acute and chronic manifestations, with progressive organ damage and vasculopathy [2]. More specifically, deoxygenation leads to the formation of polymers of haemoglobin S (HbS) that causes red blood cells (RBCs) sickling and increases their adherence to the endothelium, resulting in vaso-occlusion and tissue hypoxia [2]. These mechanisms are the basis of vaso-occlusive pain episodes (VOEs), as well as contribute to the pathogenesis of acute chest syndrome (ACS) and other major complications [3].

Sleep-disordered breathing (SDB) with nocturnal hypoxaemia, including obstructive sleep apnoea (OSA), is frequent in patients with SCD [4]. OSA is characterised by upper airway obstruction (partial or total) that disrupts ventilation, causing haemoglobin desaturation and poor sleep quality [5]. The increased prevalence of OSA and SDB in children with SCD is influenced by the high frequency of adenotonsillar hypertrophy, which is likely to be a compensatory mechanism of the immune system to functional hyposplenism [6,7]. While it has been demonstrated that a low nocturnal haemoglobin oxygen saturation $\left(\mathrm{P}_{\mathrm{PO}_{2}}\right)$ in subjects with SCD is associated with cerebrovascular complications [8,9], most recent prospective studies did not find a significant association of low oxygen saturation with VOE and ACS $[9,10]$.

Tonsillectomy and adenoidectomy (T\&A) is recommended for children with documented OSA and adenotonsillar hypertrophy in the general paediatric population [11]. Few studies reported improved overnight oxygenation in children with SCD and SDB undergoing T\&A [12-14]; FArRell et al. [12] also showed a reduction in the hospital admission rate after T\&A. However, the amount of available data regarding the effect of T\&A on the clinical course of SCD is still limited and further investigation is required.

This study tested the hypothesis that children with SCD undergoing T\&A for obstructive SDB/OSA with severe adenotonsillar hypertrophy had improved overnight oxygen saturation and decreased hospitalisation rates for ACS and VOEs following the surgery.

\section{Materials and methods}

Medical records of children with SCD from two major tertiary hospitals (Evelina London Children's Hospital and King's College Hospital, London, UK), who underwent T\&A between 2008 and 2014, were retrospectively evaluated. Data were collected within clinical audit report framework and routine quality improvement analysis. Patients who had comparable overnight oximetry studies (TCM 4/40 monitoring system, Radiometer, software version 3.0, $\mathrm{S}_{\mathrm{pO}_{2}}$ averaging time $3 \mathrm{~s}$ ) performed at steady state within 18 months before and after the surgery, were included. Nocturnal oximetry was carried out at Evelina London Children's Hospital sleep laboratory over a single night, allowing the accompanying parent to stay in the same room with the child and a sleep diary was recorded. Artefacts due to poor perfusion, low signal identification and movement were manually excluded, as well as periods of wakefulness according to the sleep diary's records. Oximetry tests with less than $4 \mathrm{~h}$ of artefact-free data were excluded. Analysis software provided standard parameters including overnight mean and nadir $S_{\mathrm{pO}_{2}}, 3 \%$ oxygen desaturation index (3\% ODI) number of validated desaturations of at least $3 \%$ per hour of sleep). In each participant the nocturnal oximetry tests performed more closely to the surgery were compared. The presence of SDB was established according to reported typical symptoms (e.g. loud snoring, witnessed apnoeas, restless sleep and mouth breathing). The proportion of patients with a $3 \% \mathrm{ODI} \geqslant 3$ at each data point was recorded, as the combination of SDB and $3 \%$ $\mathrm{ODI} \geqslant 3$ was shown to be highly predictive of OSA in a retrospective cohort of paediatric patients [15]. Information on participants' demographics, past medical history and peri-operative complications were also taken from medical records. ACS was defined as the presence of fever and/or respiratory symptoms, plus a new pulmonary infiltrate on a chest radiography [16], whereas a VOE was identified as an episode of acute pain that lasted at least $4 \mathrm{~h}$ for which there was no explanation other than vaso-occlusion and that required hospital admission with administration of parenteral opioids or nonsteroidal anti-inflammatory drugs [16].

The number of VOE and ACS episodes requiring hospitalisation and the total number of hospitalisations in the 12 months before and after T\&A were recorded in each patient.

Patients who started hydroxyurea (HU) therapy or chronic transfusion regimen in the 12 months before and after T\&A were excluded as these factors could affect the incidence of VOE and ACS [17], acting as confounders in our analysis on of the impact of T\&A on clinical outcomes. However, patients who had been on $\mathrm{HU}$ or chronic transfusion for least 12 months prior to surgery were retained in the analysis as, in this case, there was no other intervention, apart from T\&A, introduced during the study period.

\section{Statistical analysis}

Continuous variables were expressed as mean or median (interquartile range). The mean annual rates of VOE and ACS were defined as the average number of events per patient per year requiring hospital 
admission and were calculated for the year preceding and following the surgery. The mean annual rate of "hospital admissions for all causes" was also calculated over the same time interval. All variables were analysed for normality distribution (D'Agostino-Pearson omnibus normality test). Differences between groups were evaluated by Wilcoxon matched-pairs signed-rank test ( $3 \%$ ODI, \% time spent $S_{\mathrm{pO}_{2}}<90 \%$ ) and paired t-test (overnight mean $S_{\mathrm{pO}_{2}}$, nadir $S_{\mathrm{pO}_{2}}$, mean number of VOE, ACS and total hospitalisations per patient per year). Fisher's exact test was used to compare frequencies and percentages. A p-value $\leqslant 0.05$ was considered statistically significant. Statistical analyses were performed using GraphPad Prism version 8.4.2 for Windows (GraphPad Software, La Jolla, CA, USA; www.graphpad.com).

\section{Results}

\section{General characteristics of the study population}

47 children with SCD underwent T\&A between 2008 and 2014. After excluding 7 patients who started HU during the follow-up period, 3 who were treated with supplementary oxygen treatment at home in the year after surgery because of persistent hypoxaemia and other 18 patients who had no comparable nocturnal oximetry tests before and after T\&A, 19 (10 males, 53\%) children (17 HbSS, 89.5\%; 2 HbSC, 10.5\%) were retained in the final analysis. Median age at surgery was 6 years (range 3.5-8). Three (16\%) out of 19 children were already on HU before undergoing T\&A (HU started at least 1 year prior to the surgery), while 1 (5\%) out of 19 was started on exchange blood transfusion (EBT) 17 months before, because of abnormal transcranial doppler velocities. One child (5\%) had adenoidectomy alone, while 18 (95\%) out of 19 underwent T\&A. Indication to T\&A was based in most cases (15 (79\%) out of 19) on the presence of SDB symptoms with a $3 \%$ ODI $\geqslant 3$ [15]. In a minority of children T\&A was performed for severe adenotonsillar hypertrophy with SDB symptoms according to the indication of the ear, nose and throat specialist $(2(10.5 \%)$ out of 19$)$. Finally, in two children $(10.5 \%)$ it was not possible to establish with certainty from medical records the indication to T\&A because of missing data.

All participants received a pre-operative blood transfusion in order to achieve a target $\mathrm{Hb}$ level of 90-100 g.L $\mathrm{L}^{-1}$ [18]. One child presented with acute upper airway obstruction post-operatively and required a 48-h admission in the paediatric intensive care unit. No other intra-operative and post-operative major complications were reported. Other baseline features are shown in table 1.

\section{Nocturnal oximetry before and after T\&A}

Night-time oximetry studies were recorded at a median time interval of 5.5 months before (range 4.5-10.5) and 7.5 months after the surgery (range 6.3-10.8) (table 2).

\section{TABLE 1 Baseline characteristics of 19 patients with sickle cell disease who underwent}

\section{tonsillectomy and adenoidectomy}

\section{Variable}

$\begin{array}{lc}\text { Patients n } & 19 \\ \text { Males } & 10(53 \%) \\ \text { Age at surgery years } & 6(3.5-8) \\ \text { Hb type } & 17(89.5 \%) \\ \text { SS } & 2(10.5 \%) \\ \text { SC } & 3(16 \%) \\ \text { HU } & 1(5 \%) \\ \text { EBT } & 3(16 \%) \\ \text { Asthma } & \\ \text { Tonsil enlargement grading" } & \\ \quad \text { None } & 2(10.5 \%) \\ \text { Grade I } & 1(5 \%) \\ \text { Grade II } & 2(10.5 \%) \\ \text { Grade III } & 7(37 \%) \\ \text { Grade IV } & 7(37 \%) \\ \text { Snoring } \geqslant 3 \text { times a week } & + \\ \end{array}$

Data are presented as $\mathrm{n}(\%)$ or median (interquartile range), unless otherwise stated. $\mathrm{Hb}$ : haemoglobin $\mathrm{HU}$ : hydroxyurea; EBT: exchange blood transfusion. \#: Diagnosis was made in compliance with Global Initiative for Asthma guidelines [19]; ๆ: according to tonsil grading scale by Brodsky [34]; ${ }^{+}$: according to the current definition of snoring in sleep-disordered breathing [20]. 
TABLE 2 Effect of tonsillectomy and adenoidectomy (T\&A on overnight oximetry $\left(\mathrm{S}_{\mathrm{pO}_{2}}\right)$ of children with sickle cell disease $(n=19)$

\begin{tabular}{lccc} 
& Before T\&A & After T\&A & p-value \\
\hline Overnight $S_{\mathrm{po}_{2}} \%^{\#}$ & $93.0 \pm 3.6$ & $95.3 \pm 2.8$ & $\mathbf{0 . 0 0 1}$ \\
Nadir overnight $S_{\mathrm{po}_{2}} \%{ }^{\#}$ & $83.0 \pm 7.1$ & $88 \pm 4.1$ & $\mathbf{0 . 0 0 4}$ \\
$\%$ time $\mathrm{S}_{\mathrm{pO}_{2}<90 \%{ }^{7}}$ & $0.3(0-6)$ & $0.1(0-2)$ & 0.107 \\
Nocturnal 3\% ODI" & $5.7(3.6-7.8)$ & $1.8(1.2-6.0)$ & $\mathbf{0 . 0 0 3}$ \\
\hline
\end{tabular}

Data are presented as mean \pm SD or median (interquartile range), unless otherwise stated. \#: Passed the normality test: analysed by paired t-test; १: not passed the normality test: analysed by Wilcoxon matched-pairs signed-rank test. ODI: oxygen desaturation index.

After T\&A, there was a significant increase of mean overnight $S_{\mathrm{pO}_{2}}(\mathrm{p}=0.001)$ and nadir $S_{\mathrm{pO}_{2}}(\mathrm{p}=0.004)$, as well as an improvement of $3 \%$ ODI $(\mathrm{p}=0.003)$ (table 2$)$. In children with SDB and $3 \%$ ODI $\geqslant 3(15(79 \%)$ out of 19), improvements in overnight oxygen saturation parameters were comparable to those of the whole sample (data not shown). The proportion of patients with a $3 \%$ ODI $\geqslant 3$ at nocturnal oximetry passed from $79 \%$ (15 out of 19$)$ to $37 \%$ (7 out of 19$)(\mathrm{p}=0.02)$, whereas that of children with a mean overnight oximetry $<94 \%$ decreased from $42 \%$ ( 8 out of 19$)$ to $26 \%$ ( 5 out of 19$)$ ( $p=0.49$ ).

\section{SCD-related complications before and after T\&A}

Following the surgery, the mean annual rate of ACS dropped from $0.6 \pm 1.22$ to $0.1 \pm 0.2$ per patient per year ( $p=0.03$ ), while the mean cumulative rate of hospitalisation from every cause and the incidence of hospital admissions for VOE were not affected (figure 1).

\section{Discussion}

In this study, for the first time, we showed that children with SCD who underwent T\&A had significantly reduced rates of ACS episodes. Hospital admissions from all causes did not significantly change at 1-year follow-up after the surgery. We also report that children with SCD who underwent T\&A had improved overnight $S_{\mathrm{pO} 2}$, as previously described $[12,13]$.

Sleep abnormalities are characterised by intermittent hypoxia that may increase oxidative stress activating inflammatory pathways $[21,22]$. Moreover, oxygen desaturation promotes $\mathrm{HbS}$ polymerisation and RBC sickling, which in turn causes microvascular occlusion, inflammation, haemolysis and tissue hypoxia, potentially triggering SCD-related acute and chronic manifestations [23]. Given these factors, the increase in SCD severity would be expected in patients with SDB and nocturnal hypoxaemia. However, some previous studies only showed an association between nocturnal hypoxaemia and central nervous system complications $[8,24]$, whereas others failed to demonstrate an increased risk of ACS [9] and VOE $[9,10]$ in relation to a low night-time $S_{\mathrm{pO}_{2}}$.

Our results support the hypothesis that T\&A improves overnight blood oxygenation and intermittent oxygen desaturation in children with SCD and upper airway obstruction, consistently with previous

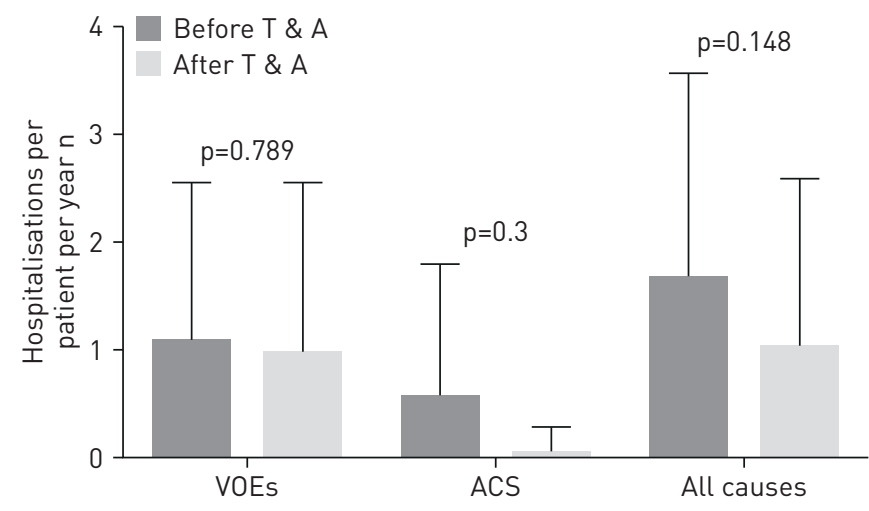

FIGURE 1 Mean annual rates of hospital admission for vaso-occlusive pain episodes (VOEs), acute chest syndrome (ACS) and for "all causes" in 19 children with sickle cell disease during the 12 months before and after tonsillectomy and adenoidectomy (T\&A). Groups were compared through a paired t-test. 
retrospective analysis [12-14, 25]. In particular, significant reduction of snoring and daytime somnolence, with reported better school attendance and objective improvement in sleep study parameters were previously observed after T\&A in a small cohort of eight children with upper airway obstruction and SCD [25]. Our study showed for the first time that T\&A was associated with a significant reduction in the number of ACS events per patient. $\mathrm{yr}^{-1}$. Previously, $\mathrm{FINCH}$ et al. [13] did not find a reduced incidence of ACS and VOE in children with SCD after T\&A, but only 13 children were evaluated, fewer than in our cohort. More recently, FARrell et al. [12] observed a drop in the rate of overall emergency department visits per patient per year in the 12 months following T\&A. In our study we did not recorded unscheduled emergency department visits, but this intervention did not result in a significant reduction of hospital admission rate. Moreover, contrary to the present study, FARRELL et al. [12] reported that ACS had a similar incidence pre- and post-surgery. In the presence of low alveolar oxygen tension, abnormal sickle RBC tend to stick together and to adhere to leukocytes and vascular endothelium. This process causes vaso-occlusion, inflammation and, in the pulmonary circulation, it may cause hypoxic vasoconstriction [26]. As these mechanisms are among the triggers of ACS episodes [27], we hypothesised that the relative increase in blood oxygenation that occurred in our cohort after T\&A, had a protective role against RBC sickling in the pulmonary microcirculation, contributing to prevent ACS. Further verification on prospective longitudinal data or randomised controlled trials, with a sample specifically powered for this outcome (the impact of T\&A on ACS incidence) and a longer follow-up, is warranted.

In non-SCD children with OSA it was shown that the restoration of an adequate and stable overnight $S_{\mathrm{pO}_{2}}$ may also prevent the risk of cardiovascular complications, neurocognitive impairment and poor school performance $[28,29]$. The reduction of ODI after T\&A seen in our SCD cohort, therefore, might result in a decreased likelihood of future OSA-related morbidities.

The present study confirmed recently reported low rates of peri-operative complications in children with SCD undergoing T\&A [12]. In general, surgery in SCD patients implies a significantly increased risk due to anaemia, hypoxia and possible post-surgical bleeding [30, 31]. However, since 1995 pre-operative transfusion protocols have been largely implemented with a dramatic decrease of post-surgical complications [32-35]. Currently, blood transfusion is universally recommended for SCD patients with low $\mathrm{Hb}$ level $\left(<90 \mathrm{~g} \cdot \mathrm{L}^{-1}\right)$ undergoing a mild to moderately severe surgical procedure and/or general anaesthesia [18]. Accordingly, all our children had an elective blood transfusion prior to T\&A to increase $\mathrm{Hb}$ level to a target level of $90-100 \mathrm{~g} \cdot \mathrm{L}^{-1}$ [18]. The low frequency of peri-operative complications indicates that T\&A has a good safety profile in children with SCD when performed in centres experienced in the management of this condition.

Among the limitations of this study, because complete polysomnography results, including apnoea-hypopnoea index (AHI), were available only in a minority of patients (5 (26\%) out of 19), we could not properly identify children with OSA, even if the combination of SDB symptoms and 3\% ODI $\geqslant 3$ (that was the main indication to T\&A in our cohort of SCD patients) was shown to be highly predictive of an $\mathrm{AHI}>5$ in children [15]. However, as the use of $3 \% \mathrm{ODI} \geqslant 3$ in combination with SDB to define OSA came from a cohort of otherwise healthy paediatric patients, this criterion may lead to a misinterpretation of the results, as SCD children have a higher prevalence of hypoxaemia than healthy children, and several studies suggested that this condition may not be due to OSA alone [36]. This might also explain why the $3 \%$ ODI did not improve in all patients after T\&A.

Moreover, nocturnal hypoxia may also be present in several major cardiovascular complications of SCD, including pulmonary hypertension and higher end-diastoclic left ventricular diameters [37]. As per the recommendations [38], children with SCD usually undergo routine echocardiography screening every 1-3 years in the absence of further indication, and in our cohort none of the enrolled patients developed any cardiac complications within the studied period. However, due to the retrospective design of this study, it was not possible to specifically compare pre- and post-echocardiography measures.

We excluded SCD patients who were started on HU or supplemental oxygen therapy during the post-surgery follow-up, as it was not possible to control for these important confounders. However, by doing so, our sample became less representative of the general SCD population, where most severe patients are usually initiated on HU.

A strength of this study was that, despite the retrospective design, we could compare pre- and post-T\&A overnight oximetry data that were collected when patients were at steady state, with tests performed in the same environment, using the same methodology and device. Moreover, the availability of detailed digitalised medical history information for each patient allowed a precise measure of the incidence of SCD-related complications and overnight oximetry in the year prior to and after T\&A. 
In summary, this study showed that children with SCD who have SDB and nocturnal hypoxaemia may benefit from T\&A in terms of improved overnight $S_{\mathrm{pO}_{2}}$ and decreased incidence of ACS episodes. Moreover, our study confirmed that T\&A in paediatric patients with SCD has a satisfactory safety profile when performed in experienced centres. Future studies should further clarify the impact of T\&A on the incidence of SCD-related complications in prospective cohorts with a longer follow-up and adequate control for confounding.

Acknowledgment: The authors are grateful to the Paediatric ENT team at Evelina London Children's Hospital, to the Paediatric Sleep teams at Evelina London Children's Hospital and King's College Hospital, to the Paediatric Sickle Cell Disease teams at Evelina London Children's Hospital and King's College Hospital and to the Paediatric Respiratory team at King's College Hospital. Finally, the authors also thank all the patients and their parents.

Conflict of interest: I. Liguoro has nothing to disclose. M. Arigliani has nothing to disclose. B. Singh has nothing to disclose. L. Van Geyzel has nothing to disclose. S. Chakravorty has nothing to disclose. C. Bossley has nothing to disclose. M. Pelidis has nothing to disclose. D. Rees has nothing to disclose. B.P.D. Inusa reports educational grants from Novartis plc, AstraZeneca, Cyclerion and Bluebird bio, outside the submitted work. A. Gupta has nothing to disclose.

\section{References}

1 Piel FB, Patil AP, Howes RE, et al. Global epidemiology of sickle haemoglobin in neonates: a contemporary geostatistical model-based map and population estimates. Lancet 2013; 381: 142-151.

2 Rees DC, Williams TN, Gladwin MT. Sickle-cell disease. Lancet 2010; 376: 2018-2031.

3 Liberto GD, Kiger L, Marden MC, et al. Dense red blood cell and oxygen desaturation in sickle-cell disease. Am J Hematol 2016; 91: 1008-1013.

4 Rosen CL, Debaun MR, Strunk RC, et al. Obstructive sleep apnea and sickle cell anemia. Pediatrics 2014; 134: 273-281.

5 Section on Pediatric Pulmonology, Subcommittee on Obstructive Sleep Apnea Syndrome. American Academy of Pediatrics. Clinical practice guideline: diagnosis and management of childhood obstructive sleep apnea syndrome. Pediatrics 2002; 109: 704-712.

6 Salles C, Ramos RTT, Daltro C, et al. Association between adenotonsillar hypertrophy, tonsillitis and painful crises in sickle cell disease. J Pediatr (Rio J) 2009; 85: 249-253.

7 Strauss T, Sin S, Marcus CL, et al. Upper airway lymphoid tissue size in children with sickle cell disease. Chest 2012; 142: 94-100.

8 Dlamini N, Saunders DE, Bynevelt M, et al. Nocturnal oxyhemoglobin desaturation and arteriopathy in a pediatric sickle cell disease cohort. Neurology 2017; 89: 2406-2412.

9 Halphen I, Elie C, Brousse V, et al. Severe nocturnal and postexercise hypoxia in children and adolescents with sickle cell disease. PLoS ONE 2014; 9: e97462.

10 Willen SM, Rodeghier M, Rosen CL, et al. Sleep disordered breathing does not predict acute severe pain episodes in children with sickle cell anemia. Am J Hematol 2018; 93: 478-485.

11 Mitchell RB, Archer SM, Ishman SL, et al. Clinical practice guideline: tonsillectomy in children (update) Otolaryngol Neck Surg 2019; 160: S1-S42.

12 Farrell AN, Goudy SL, Yee ME, et al. Adenotonsillectomy in children with sickle cell disease and obstructive sleep apnea. Int J Pediatr Otorhinolaryngol 2018; 111: 158-161.

13 Finch P, Stocks RM, Smeltzer MP, et al. Effects of adenotonsillectomy on polysomnographic parameters in children with sickle cell disease. Pediatr Blood Cancer 2013; 60: E26-E28.

14 Samuels MP, Stebbens VA, Davies SC, et al. Sleep related upper airway obstruction and hypoxaemia in sickle cell disease. Arch Dis Child 1992; 67: 925-929.

15 Chang L, Wu J, Cao L. Combination of symptoms and oxygen desaturation index in predicting childhood obstructive sleep apnea. Int J Pediatr Otorhinolaryngol 2013; 77: 365-371.

16 Ballas SK, Lieff S, Benjamin LJ, et al. Definitions of the phenotypic manifestations of sickle cell disease. Am J Hematol 2010; 85: 6-13.

17 Tshilolo L, Tomlinson G, Williams TN, et al. Hydroxyurea for children with sickle cell anemia in Sub-Saharan Africa. N Engl J Med 2019; 380: 121-131.

18 Steiner ME, Zantek ND, Stanworth SJ, et al. Recommendations on RBC transfusion support in children with hematologic and oncologic diagnoses from the Pediatric Critical Care Transfusion and Anemia Expertise Initiative. Pediatr Crit Care Med 2018; 19: S149-S156.

19 Global Initiative for Asthma. Global Strategy for Asthma Management and Prevention, 2019. Available from:. www.ginasthma.org.

20 Marcus CL, Brooks LJ, Draper KA, et al. Diagnosis and management of childhood obstructive sleep apnea syndrome. Pediatrics 2012; 130: 576-584.

21 Ryan S, Taylor CT, McNicholas WT. Selective activation of inflammatory pathways by intermittent hypoxia in obstructive sleep apnea syndrome. Circulation 2005; 112: 2660-2667.

22 Almendros I, Wang Y, Gozal D. The polymorphic and contradictory aspects of intermittent hypoxia. Am J Physiol Lung Cell Mol Physiol 2014; 307: L129-L140.

23 Caboot JB, Allen JL. Hypoxemia in sickle cell disease: significance and management. Paediatr Respir Rev 2014; 15 $17-23$.

24 Kirkham F, Hewes D, Prengler M, et al. Nocturnal hypoxaemia and central-nervous-system events in sickle-cell disease. Lancet 2001; 357: 1656-1659.

25 Warrier R, Chauhan A, Athale U. Tonsillectomy and adenoidectomy for obstructive sleep apnea in sickle cell anemia. Indian J Pediatr 2010; 77: 669-672.

26 Platt OS. Sickle cell anemia as an inflammatory disease. J Clin Invest 2000; 106: 337-338. 
27 Gladwin MT, Rodgers GP. Pathogenesis and treatment of acute chest syndrome of sickle-cell anaemia. Lancet Lond Engl 2000; 355: 1476-1478.

28 Dehlink E, Tan H-L. Update on paediatric obstructive sleep apnoea. J Thorac Dis 2016; 8: 224-235.

29 Biggs SN, Vlahandonis A, Anderson V, et al. Long-term changes in neurocognition and behavior following treatment of sleep disordered breathing in school-aged children. Sleep 2014; 37: 77-84.

30 Maddern BR, Ohene-Frempong K, Reed HT, et al. Obstructive sleep apnea syndrome in sickle cell disease. Ann Otol Rhinol Laryngol 1989; 98: 174-178.

31 Derkay CS, Bray G, Milmoe GJ, et al. Adenotonsillectomy in children with sickle cell disease. South Med J 1991; 84: 205-208.

32 Vichinsky EP, Haberkern CM, Neumayr L, et al. A comparison of conservative and aggressive transfusion regimens in the perioperative management of sickle cell disease. The Preoperative Transfusion in Sickle Cell Disease Study Group. N Engl J Med 1995; 333: 206-213.

33 Waldron P, Pegelow C, Neumayr L, et al. Tonsillectomy, adenoidectomy, and myringotomy in sickle cell disease: perioperative morbidity. Preoperative Transfusion in Sickle Cell Disease Study Group. J Pediatr Hematol Oncol 1999; 21: 129-135.

34 Wali YA, al Okbi H, al Abri R. A comparison of two transfusion regimens in the perioperative management of children with sickle cell disease undergoing adenotonsillectomy. Pediatr Hematol Oncol 2003; 20: 7-13.

35 Duke R, Scott J, Panepinto J, et al. Perioperative management of sickle cell disease children undergoing adenotonsillectomy. Otolaryngol Head Neck Surg 2006; 134: 370-373.

36 Needleman JP, Franco ME, Varlotta L, et al. Mechanisms of nocturnal oxyhemoglobin desaturation in children and adolescents with sickle cell disease. Pediatr Pulmonol 1999; 28: 418-422.

37 Mondal P, Stefek B, Sinharoy A, et al. The association of nocturnal hypoxia and an echocardiographic measure of pulmonary hypertension in children with sickle cell disease. Pediatr Res 2019; 85: 506-510.

38 Liem RI, Lanzkron S, Coates T D, et al. American Society of Hematology 2019 guidelines for sickle cell disease: cardiopulmonary and kidney disease. Blood Adv 2019; 3: 3867-3897. 